\title{
Dual role of chloroquine in liver ischemia reperfusion injury: reduction of liver damage in early phase, but aggravation in late phase
}

\author{
H Fang $^{1,3,5}$, A Liü ${ }^{2,3,5}$, U Dahmen ${ }^{\star, 3}$ and O Dirsch ${ }^{4}$
}

The anti-malaria drug chloroquine is well known as autophagy inhibitor. Chloroquine has also been used as anti-inflammatory drugs to treat inflammatory diseases. We hypothesized that chloroquine could have a dual effect in liver ischemia/reperfusion (I/R) injury: chloroquine on the one hand could protect the liver against I/R injury via inhibition of inflammatory response, but on the other hand could aggravate liver I/R injury through inhibition of autophagy. Rats ( $n=6$ per group) were pre-treated with chloroquine (60 mg/kg, i.p.) $1 \mathrm{~h}$ before warm ischemia, and they were continuously subjected to a daily chloroquine injection for up to 2 days. Rats were killed $0.5,6,24$ and $48 \mathrm{~h}$ after reperfusion. At the early phase (i.e., $0-6 \mathrm{~h}$ after reperfusion), chloroquine treatment ameliorated liver I/R injury, as indicated by lower serum aminotransferase levels, lower hepatic inflammatory cytokines and fewer histopathologic changes. In contrast, chloroquine worsened liver injury at the late phase of reperfusion (i.e., 24-48 $\mathrm{h}$ after reperfusion). The mechanism of protective action of chloroquine appeared to involve its ability to modulate mitogen-activated protein kinase activation, reduce high-mobility group box 1 release and inflammatory cytokines production, whereas chloroquine worsened liver injury via inhibition of autophagy and induction of hepatic apoptosis at the late phase. In conclusion, chloroquine prevents ischemic liver damage at the early phase, but aggravates liver damage at the late phase in liver I/R injury. This dual role of chloroquine should be considered when using chloroquine as an inhibitor of inflammation or autophagy in I/R injury.

Cell Death and Disease (2013) 4, e694; doi:10.1038/cddis.2013.225; published online 27 June 2013

Subject Category: Experimental Medicine

Ischemia/reperfusion (I/R) injury occurs during the procedure of liver transplantation, liver resection, trauma and other surgical procedures when the liver is transiently deprived of oxygen and subsequently re-oxygenated. ${ }^{1,2}$ Injury to the liver caused by I/R has been shown be associated with increased rate of acute liver failure/graft rejection and chronic liver dysfunction after liver transplantation.

The mechanism of liver damage after I/R has been studied extensively. The signaling events contributing to cellular and tissue damage are diverse and complex, and consist of complex interactions of multiple inflammatory pathways. ${ }^{3-5}$ Proinflammatory cytokines, such as high-mobility group box 1 (HMGB1), tumor necrosis factor (TNF)- $\alpha$ and interleukin (IL)-1 $\beta$ are known to increase in serum and liver tissue on reperfusion and have a pivotal role in the pathophysiology of hepatic I/R injury. ${ }^{6-9}$ Chloroquine, a well-known anti-malaria drug, has also been frequently used in the treatment of inflammatory diseases, including rheumatoid arthritis and systemic lupus erythematodes. ${ }^{10}$ It has been suggested that the therapeutic effect of chloroquine in these diseases is related to its immunoregulatory potency. Recent evidences have shown that chloroquine inhibits proinflammatory cytokine production by macrophages or monocytes after stimulation with lipopolysaccharide (LPS) or CpG oligonucleotide (CpG ODN). ${ }^{11-13}$ In vivo, chloroquine protected mice from lethal doses of LPS or CpG ODN through a decrease of proinflammatory cytokine release. ${ }^{11}$ Furthermore, chloroquine protected mice from sepsis-induced acute kidney injury and decreased serum inflammatory cytokines levels. ${ }^{14}$ Prompted by these results, we hypothesized that chloroquine could also possess anti-inflammatory abilities to protect from or reduce hepatic I/R injury, which is characterized as inflammatory injury.

In contrast to its ability to inhibit inflammation, there is a growing body of evidence suggesting that chloroquine

\footnotetext{
${ }^{1}$ Department of Pathophysiology, Anhui Medical University, Hefei 230032, China; ${ }^{2}$ Experimental Medicine Center, Tongji Hospital, Tongji Medical College, Huazhong University of Science and Technology, Wuhan 430030, China; ${ }^{3}$ Experimental Transplantation Surgery, Department of General, Visceral and Vascular Surgery, FriedrichSchiller-University Jena, Jena 07747, Germany and ${ }^{4}$ Institute for Pathology, University Hospital of Jena, Jena 07747, Germany

${ }^{*}$ Corresponding author: U Dahmen, Experimental Transplantation Surgery, Department of General, Visceral and Vascular Surgery, Friedrich-Schiller-University Jena, Drackendorfer Straße 1, Jena 07747, Germany. Tel: + 4903641932 5350; Fax: + 4903641932 5352. E-mail: uta.dahmen@med.uni-jena.de

${ }^{5}$ These authors contributed equally to this work.

Keywords: chloroquine; liver I/R injury; autophagy; apoptosis; HMGB1

Abbreviations: I/R, ischemia/reperfusion; HMGB1, high-mobility group box 1; TNF, tumor necrosis factor; IL, interleukin; LPS, lipopolysaccharide; CpG ODN, CpG oligonucleotide; AST, aspartate aminotransferase; ALT, alanine transaminase; LC3, microtubule-associated protein light chain 3; ERK, extracellular signal-regulated kinase; JNK, c-Jun N-terminal kinase; GAPDH, glyceraldehyde-3-phosphate dehydrogenase; HPRT, hypoxanthine-guanine phosphoribosyltransferase; ELISA, enzyme-linked immunosorbent assay; PCR, quantitative polymerase chain reaction; iNOS, inducible nitric oxide synthase; MAP, mitogen-activated protein; MPT, mitochondrial permeability transition; Atg, autophagy-related gene

Received 02.11.12; revised 15.5.13; accepted 16.5.13; Edited by T Brunner
} 
administration worsens $\mathrm{l} / \mathrm{R}$ injury via inhibition of autophagy. ${ }^{15,16}$ Chloroquine also induced apoptosis both in vitro and in vivo via the inhibition of autophagic protein degradation. ${ }^{17-20}$ Autophagy is involved in various physiological processes, such as liver diseases, cancer and aging, but also I/R injury. ${ }^{21-24}$ During hepatic I/R injury, autophagy was induced in the liver. Induction of autophagy was associated with a pro-survival activity allowing the cell for coping with nutrient starvation and anoxia. Induction of autophagy is associated with attenuation of $\mathrm{I} / \mathrm{R}$ injury. ${ }^{21}$ These previous results led us to hypothesize that chloroquine could also worsen liver injury via inhibition of hepatic autophagy and induction of hepatic apoptosis after I/R injury.

Given that inhibition of inflammation reduces hepatic I/R injury, and that inhibition of autophagy may worsen hepatic I/R injury, we hypothesized that chloroquine, an anti-inflammatory and autophagy inhibitory drug could have a dual effect in liver I/R injury; chloroquine on the one hand could protect the liver against I/R injury via inhibition of inflammatory response, but on the other hand could aggravate liver
I/R injury through inhibition of autophagy and induction of apoptosis. Therefore, the aim of this study was to investigate the preconditioning effects of chloroquine administration on rat hepatic I/R injury.

\section{Results}

Chloroquine reduces liver injury in the early reperfusion phase, but worsens liver injury during the late reperfusion phase after hepatic ischemic injury. To determine whether chloroquine treatment could affect liver I/R injury, chloroquine was administrated to rats $1 \mathrm{~h}$ before warm ischemia followed by a daily injection for up to 2 days. Liver warm I/R resulted in significant increase in serum levels of alanine transaminase (ALT) and aspartate aminotransferase (AST). As shown in Figure 1a, following 60 min of warm ischemia, chloroquine treatment decreased I/R injury at 0.5 and $6 \mathrm{~h}$ as indicated by lower liver enzymes $(P<0.05)$. However, the serum levels of ALT and AST were much higher following 24- and 48-h reperfusion when compared
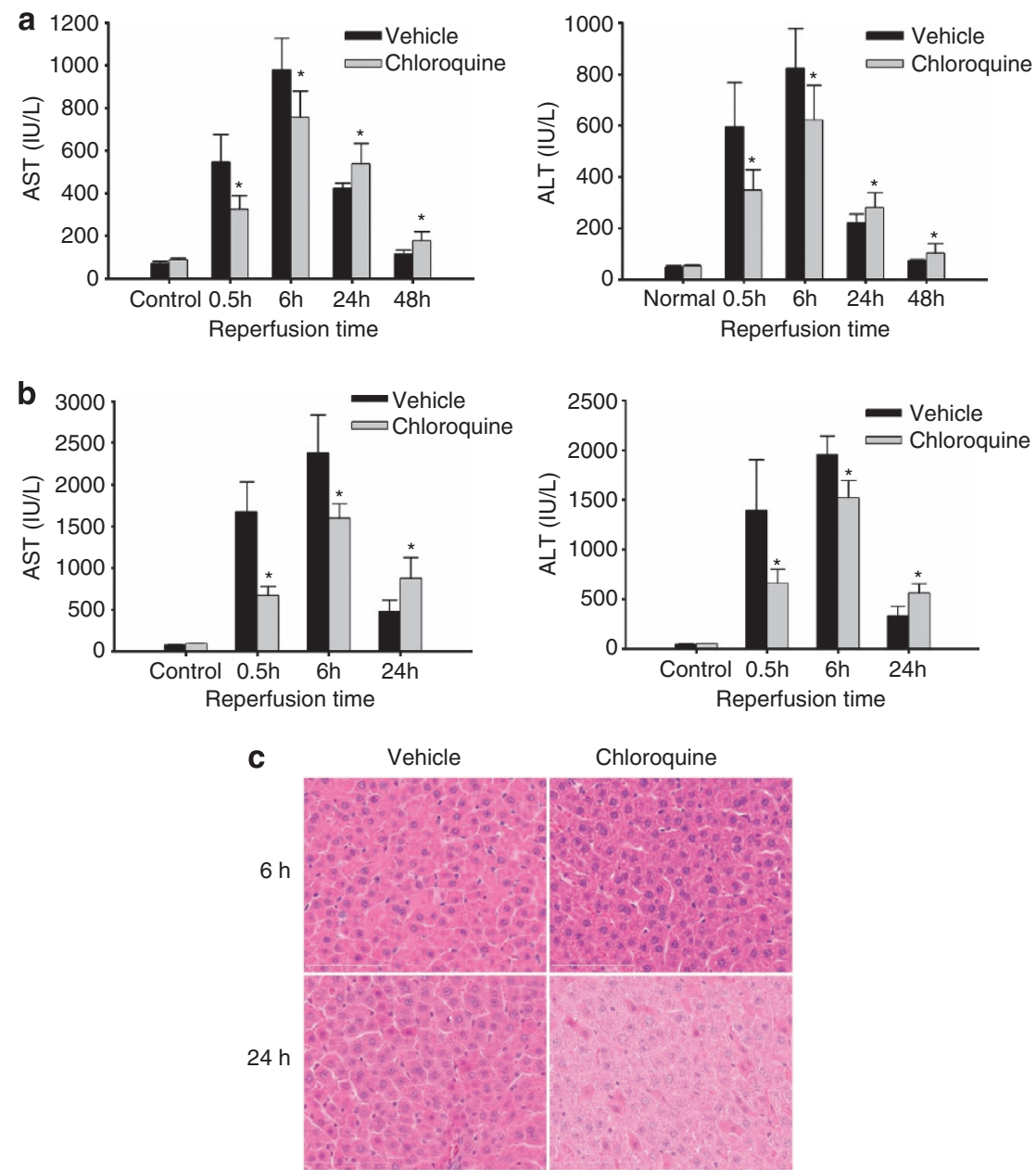

Figure 1 Chloroquine treatment prevented liver I/R injury at early phase, but worsened at late phase following I/R injury. Rats were pre-treated with either chloroquine $(60 \mathrm{mg} / \mathrm{kg}$ ) or vehicle (saline) $1 \mathrm{~h}$ before warm ischemia, followed by an additional chloroquine injections at $24 \mathrm{~h}$ after reperfusion. Following $60 \mathrm{~min}$ (a) or $90 \mathrm{~min}$ (b) of warm ischemia, liver injury was assessed at various time points following reperfusion by determination of serum AST and ALT levels. Data are shown as mean \pm S.D. ${ }^{*} P<0.05$ compared with vehicle-treated I/R group. (c) Routine histopathology was performed on formalin-fixed liver sections obtained from rats that had undergone 60 min of ischemia followed by 6 - and $24-\mathrm{h}$ reperfusion, respectively (original magnification $\times 400$ ). Representative images from six rats per group were selected 
Table 1 Histological evaluation according to the semiquantitative system

\begin{tabular}{|c|c|c|c|c|}
\hline & $\begin{array}{l}\text { Alteration } \\
\text { in } \\
\text { sinusoids }\end{array}$ & $\begin{array}{c}\text { Vacuolization } \\
\text { of } \\
\text { hepatocytes }\end{array}$ & $\begin{array}{l}\text { Erythrocyte } \\
\text { congestion }\end{array}$ & $\begin{array}{l}\text { Hepatocellular } \\
\text { necrosis }\end{array}$ \\
\hline \multicolumn{5}{|l|}{ Vehicle } \\
\hline $6 \mathrm{~h}$ & $2 \pm 1$ & $2 \pm 1$ & $2 \pm 1$ & $2 \pm 1$ \\
\hline $24 \mathrm{~h}$ & $1 \pm 0$ & $1 \pm 1$ & $1 \pm 0$ & $1 \pm 0$ \\
\hline \multicolumn{5}{|c|}{ Chloroquine } \\
\hline $6 \mathrm{~h}$ & $1 \pm 1$ & $2 \pm 1$ & $2 \pm 1$ & $1 \pm 1$ \\
\hline $24 \mathrm{~h}$ & $1 \pm 0$ & $3 \pm 1$ & $1 \pm 0$ & $1 \pm 1$ \\
\hline
\end{tabular}

with vehicle-injected rats $(P<0.05)$. To further confirm the dual effects of chloroquine on liver I/R injury, additional rats were subjected to $90 \mathrm{~min}$ of warm $\mathrm{l} / \mathrm{R}$. Chloroquine treatment caused a similar dual effect in terms of liver injury when rats were subjected to prolonged warm ischemic insult (Figure 1b).

Liver histology confirmed the findings regarding liver injury described above. Moderate hepatocellular necrosis, cytoplasmic vacuolization of hepatocytes and sinusoidal dilation were observed $6 \mathrm{~h}$ after reperfusion in vehicle-treated rats. In contrast, minor hepatocellular necrosis and slight sinusoidal dilation were observed in chloroquine treatment group. However, chloroquine-treated rats showed more severe cytoplasmic vacuolization and hepatocellular necrosis $24 \mathrm{~h}$ after reperfusion when compared with vehicle-treated rats (Figure 1c; Table 1).

Chloroquine treatment decreases hepatic inflammatory cytokines gene expression. The expression of hepatic mRNA for TNF- $\alpha$, IL-6, IL-1 $\beta$ and inducible nitric oxide synthase (iNOS) were measured by quantitative PCR. As shown in Figure 2, following 60-min warm ischemia, hepatic TNF- $\alpha$, IL-6, IL-1 $\beta$ and iNOS expression levels were increased at 0.5 and $6 \mathrm{~h}$ in both vehicle treatment group and chloroquine treatment group when compared with shamoperated controls. However, chloroquine-treated rats exhibited minimal increases in hepatic TNF- $\alpha, \mathrm{IL}-6, \mathrm{IL}-1 \beta$ and iNOS mRNA levels compared with vehicle-injected animals, accounting for about a 5-, 20-, 10- and 5-fold decrease at $6 \mathrm{~h}$ of reperfusion, respectively.

Chloroquine treatment decreases HMGB1 release at early phase. HMGB1 is a cytokine, which is released during hepatic ischemia and in the early reperfusion phase. ${ }^{8,9}$ As shown in Figure 3a, nuclear HMGB1 staining in hepatocytes was substantial loss in vehicle-injected control rats as early as $0.5 \mathrm{~h}$ after reperfusion, In contrast, pre-treatment with chloroquine largely prevents this loss of nuclear HMGB1 staining. To assess the extracellular release of HMGB1 after warm liver I/R injury, serum HMGB1 concentration was quantified using an enzyme-linked immunosorbent assay (ELISA). As shown in Figure 3b, warm I/R injury caused HMGB1 release in both vehicle-treated rats and chloroquinetreated rats. Serum levels of HMGB1 were highest at $0.5 \mathrm{~h}$ after reperfusion $(7.78 \pm 2.62 \mathrm{ng} / \mathrm{ml})$, and gradually decreased thereafter ( $6 \mathrm{~h}: 2.03 \pm 0.56$ versus normal control: $0.68 \pm 0.32 \mathrm{ng} / \mathrm{ml}, P<0.05)$. In contrast, chloroquine-treated rats showed lower serum HMGB1 levels when compared with vehicle-treated rats. The serum levels of HMGB1 were drastically decreased following reperfusion, to $1.40 \pm 0.41$, $1.28 \pm 0.45 \mathrm{ng} / \mathrm{ml}$ at 0.5 and $6 \mathrm{~h}$, respectively $(P<0.01)$.

Chloroquine treatment modulates liver I/R-induced inflammatory signaling pathways. Among the most proximal events in I/R is the activation of mitogen-activated protein (MAP) kinases. $^{25}$ To determine if chloroquine pre-treatment influenced MAP kinase activation, we assessed phosphorylation of extracellular signal-regulated kinase (ERK), c-Jun N-terminal kinase (JNK) and p38. As shown in Figure 4, after I/R, phosphorylation p38 and JNK was increased in vehicle-treated rats. In contrast, phosphorlation of JNK was decreased, whereas phosphorylation of p38 was increased in chloroquine-injected rats. Phosphorylation of ERK was increased at $0.5 \mathrm{~h}$, decreased at $6 \mathrm{~h}$ and then recovered thereafter. This dephosphorylation was partially prevented by chloroquine treatment. Treatment with chloroquine did not affect total cellular levels of JNK, p38 and ERK. These data suggest that chloroquine could act upstream of MAP kinase activation.

Chloroquine treatment decreases autophagy. Autophagy is a highly conserved cellular process that recycles damaged and or long-lived proteins and organelles. ${ }^{23,24}$ Growing evidences showed that chloroquine decreased autophagy in a number of cell types. ${ }^{26}$ To determine whether chloroquine could inhibit hepatic autophagy following I/R, western blots were performed for microtubule-associated protein light chain 3 (LC3) and p62 on livers obtained from rats pre-treated with either chloroquine or vehicle. As shown in Figure 5, shamoperated rats had low levels of LC3-II. Following $60 \mathrm{~min}$ of warm ischemia, LC3-II protein was increased at $6 \mathrm{~h}$ after reperfusion, but decreased p62 expression. In contrast, chloroquine treatment increased significantly LC3-II and p62 expression in response to liver I/R. These data indicated chloroquine inhibited hepatic post-ischemic autophagy.

\section{Chloroquine treatment increases hepatic apoptosis.} It has been reported that chloroquine-induced apoptosis both in vitro and in vivo via the inhibition of autophagic protein degradation. ${ }^{17-20}$ Prompted by these findings, we focused our studies on the expression of the apoptotic protein caspase-3 and caspase-7. As shown in Figure 6, caspase3 cleavage was increased in response to liver I/R. In contrast, chloroquine treatment increased liver I/R-induced caspase-3 cleavage. Furthermore, as with caspase-3, chloroquinetreated rats demonstrated significantly higher hepatic cleaved caspase-7 levels when compared with vehicleinjected rats. These data indicate that chloroquine treatment could increase I/R-induced apoptosis. Induction of apoptosis in the late phase of reperfusion was associated with an increased hepatic injury as indicated by the elevated liver enzyme and the severe histopathologic changes.

\section{Discussion}

The anti-malaria drug chloroquine is well known as autophagy inhibitor. The compound is also known as drug with 

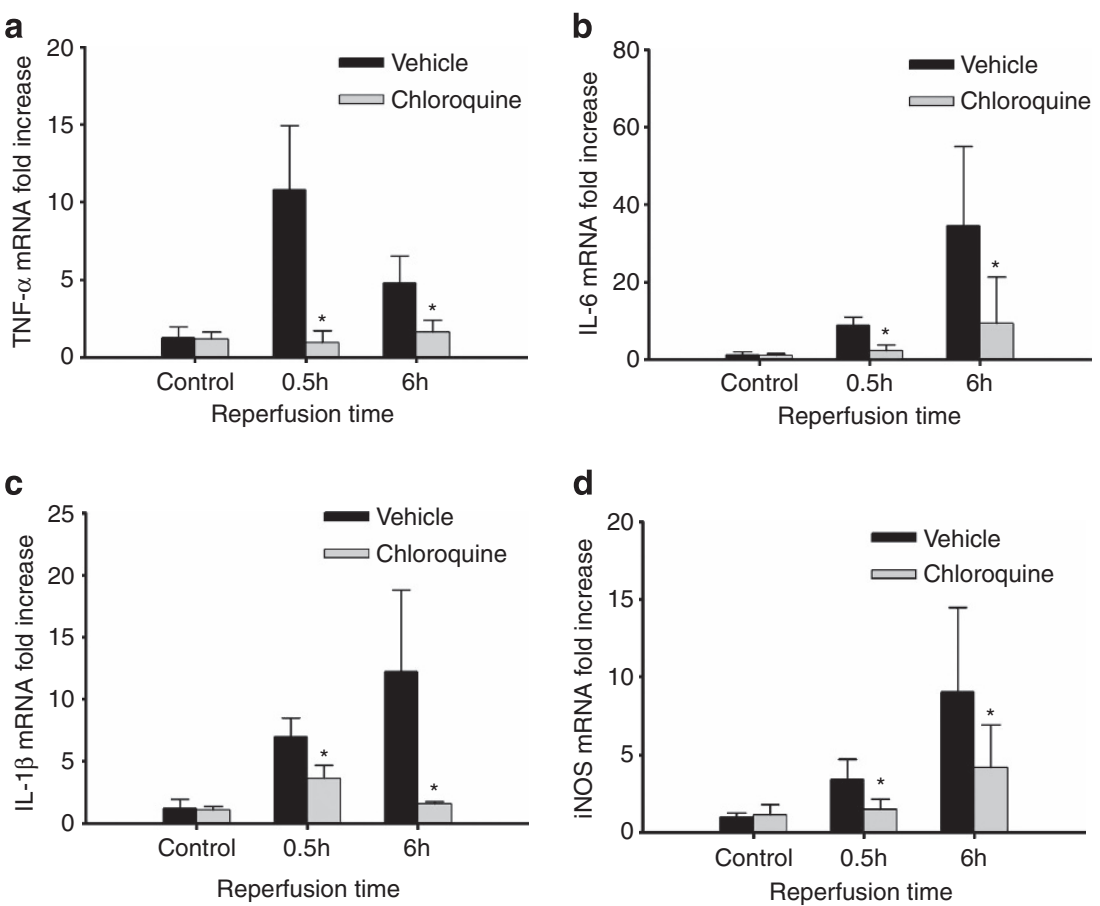

Figure 2 Chloroquine treatment decreased liver expression of inflammatory mediators at early phase following I/R injury. Rats were treated with either chloroquine $(60 \mathrm{mg} / \mathrm{kg}$ ) or vehicle (saline) $1 \mathrm{~h}$ before warm ischemia. Rats were killed 0.5 and $6 \mathrm{~h}$ after reperfusion. TNF- $\alpha$ (a), IL-6 (b), IL-1 $\beta$ (c) and iNOS (d) mRNA expression levels were measured by quantitative PCR. Results were expressed as relative increase of mRNA expression compared with normal animals. Data are shown as mean \pm S.D. $n=6$ per group. ${ }^{*} P<0.05$ compared with vehicle-treated $\mathrm{l} / \mathrm{R}$ group
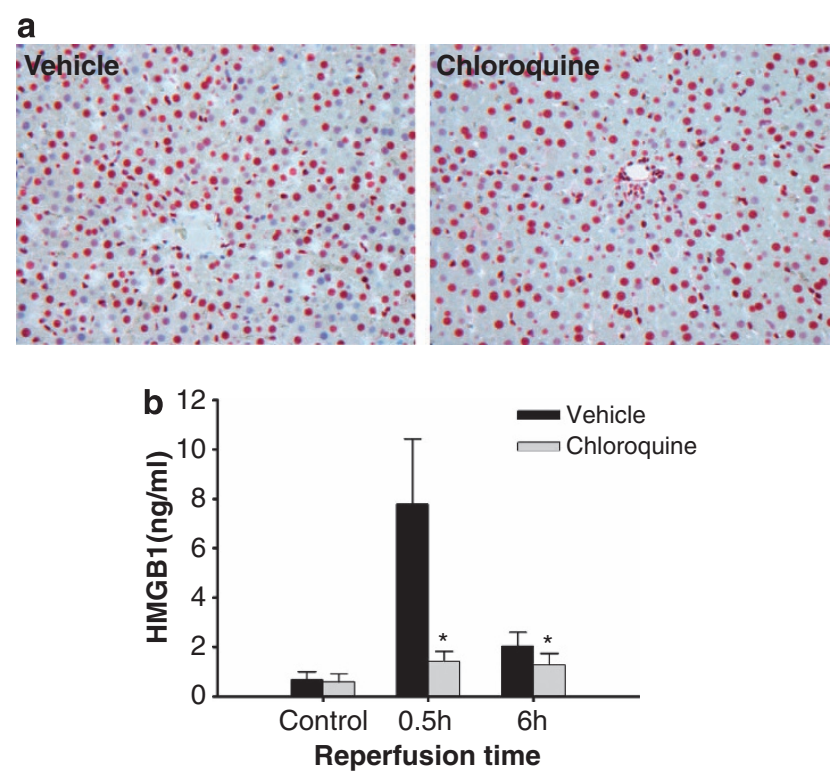

Figure 3 Chloroquine treatment decreased HMGB1 release at early phase following I/R injury. Rats were treated with either chloroquine $(60 \mathrm{mg} / \mathrm{kg})$ or vehicle (saline) $1 \mathrm{~h}$ before warm ischemia. Liver samples and serum were harvested at 0.5 and $6 \mathrm{~h}$ post-reperfusion. (a) HMGB1 cellular location was visualized by immunohistochemical staining (original magnification $\times 200$ ). Representative images from six rats per group were selected. (b) HMGB1-release into serum was quantified by ELISA. Data are shown as mean \pm S.D. $n=6$ per group. ${ }^{*} P<0.01$ compared with vehicle-treated $\mathrm{I} / \mathrm{R}$ group

anti-inflammatory properties. However, it was reported recently that chloroquine treatment aggravated I/R injury, which is characterized as inflammatory injury. ${ }^{15,16}$ This was the reason to investigate the effects of chloroquine in liver I/R injury and the underlying mechanisms in detail.

To the best of our knowledge, this is the first report showing that chloroquine has a dual effect in liver I/R injury. Following 60 or $90 \mathrm{~min}$ of warm ischemia, chloroquine treatment reduced liver injury at 0.5 and $6 \mathrm{~h}$, but aggravated liver injury at 24 and $48 \mathrm{~h}$ after reperfusion. In the early phase (i.e., 0-6 h after reperfusion), the protective effect of chloroquine treatment was associated with a modulation of MAP kinase activation, inhibition of HMGB1 release and reduction of inflammatory cytokine production. In contrast, in the late phase of reperfusion (i.e., 24-48 h after reperfusion), chloroquine treatment was associated with an aggravation of liver injury, an inhibition of autophagy and an induction of apoptosis.

To study the mechanism of protection in chloroquine treatment in the early phase after reperfusion, we investigated its effect on proinflammatory cytokines expression. Chloroquine has been extensively used in the treatment of chronic inflammatory diseases. ${ }^{27}$ We hypothesized that chloroquine may also possess anti-inflammatory abilities in liver I/R injury, which is also regarded as an inflammatory injury. To study the effects of chloroquine on proinflammatory cytokine gene expression, rats were preconditioned with chloroquine before the ischemic insult. We demonstrated that following $60 \mathrm{~min}$ of warm ischemia, chloroquine treatment reduced TNF- $\alpha$, IL-6, IL-1 $\beta$ and iNOS gene expression at 0.5 and $6 \mathrm{~h}$ after reperfusion. These cytokines are known to have a pivotal role in the pathophysiology of hepatic I/R injury. ${ }^{6,7,28}$

Recent studies suggest that extracellular HMGB1, released from damaged liver cells, causes a proinflammatory 


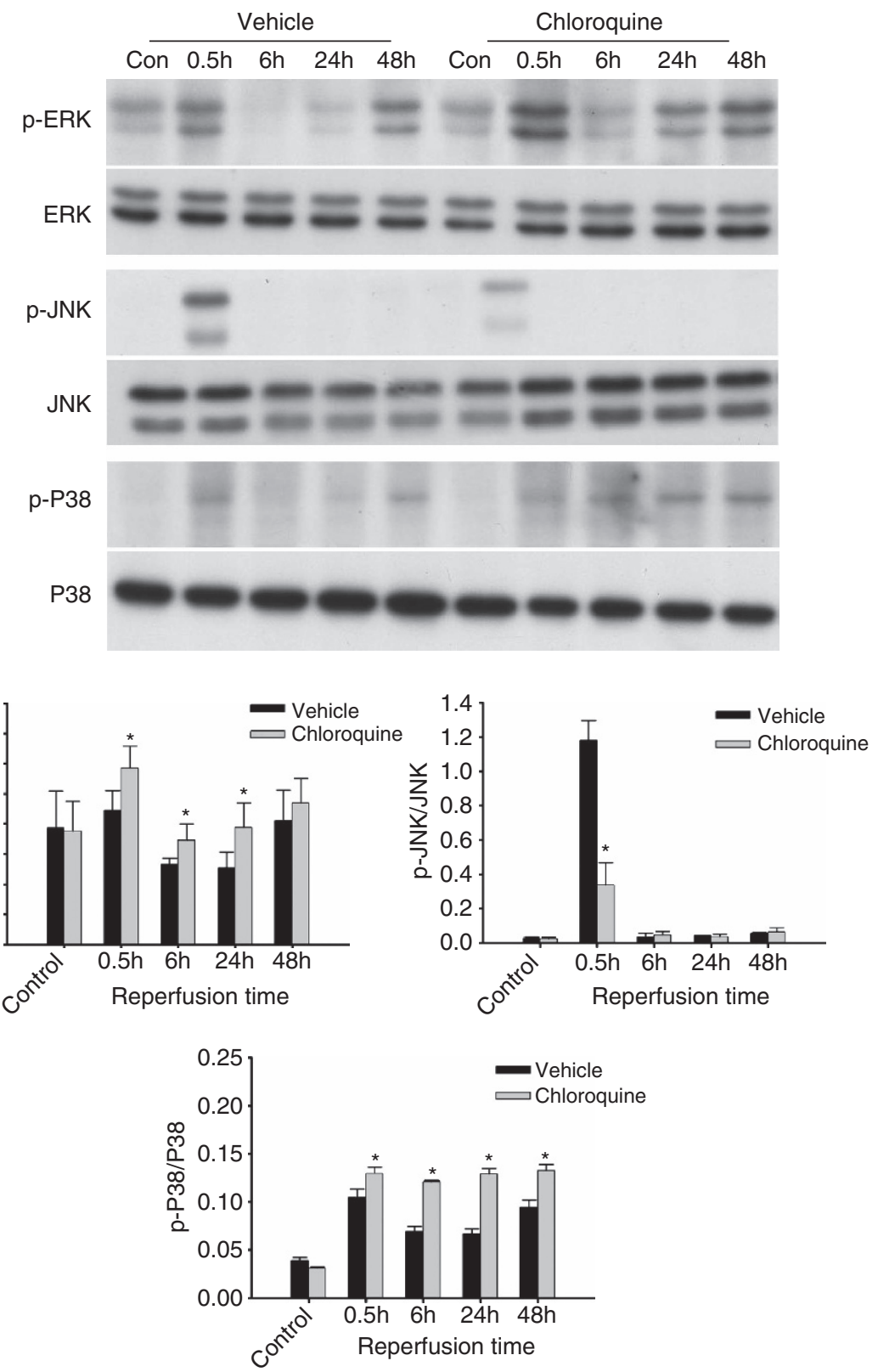

Figure 4 Chloroquine treatment modulated MAP kinase activation. Rats were pre-treated with chloroquine $(60 \mathrm{mg} / \mathrm{kg}) 1 \mathrm{~h}$ before warm ischemia, and they were continuously subjected to a daily chloroquine injection for up to 2 days. (a) Western blot analysis for phosphorylation and total ERK, JNK and p38 was performed in the ischemic lobes at various time points following reperfusion. (b) The gray value of bands was calculated by Image J. Data are shown as mean \pm S.D. $n=6$ per group. ${ }^{*} P<0.05$ compared with vehicle-treated I/R group

intra-hepatic micro-environment in the post-ischemic liver. Therefore, we investigated whether chloroquine treatment could modulate the release of HMGB1. Consistent with the findings in our previous studies and from other groups, HMGB1 was mainly present in the nucleus of hepatocytes in normal livers, and translocated from nucleus to cytoplasm on liver I/R injury. ${ }^{8,9,29,30}$ Chloroquine treatment reduced hepatic HMGB1 translocation and serum levels in the early phase. HMGB1 release induced the synthesis of proinflammatory cytokines and caused further liver damage in liver after $\mathrm{I} / \mathrm{R}$ injury. ${ }^{8,9}$ Treatment with a neutralizing antibody to HMGB1 significantly decreased liver I/R injury. ${ }^{9}$ These data suggest that the inhibition of hepatic HMGB1 translocation and subsequent release is involved in the hepatoprotective effect of chloroquine in the early phase of reperfusion.

To gain further insight into the mechanisms inducing the protective effect of chloroquine, its influence on the activation of MAPK signaling pathways was studied. It was recently demonstrated that the activation of MAP kinase pathways have a major role in liver $\mathrm{I} / \mathrm{R}$ injury. ${ }^{25}$ Chloroquine inhibits these signaling pathways in monocytes, macrophages and epithelial cells. ${ }^{13,31,32}$ Chloroquine inhibited the activation of p38 and ERK, leading to the suppression of cytokine production caused by CpG DNA. ${ }^{13,32}$ In this study, we 
a

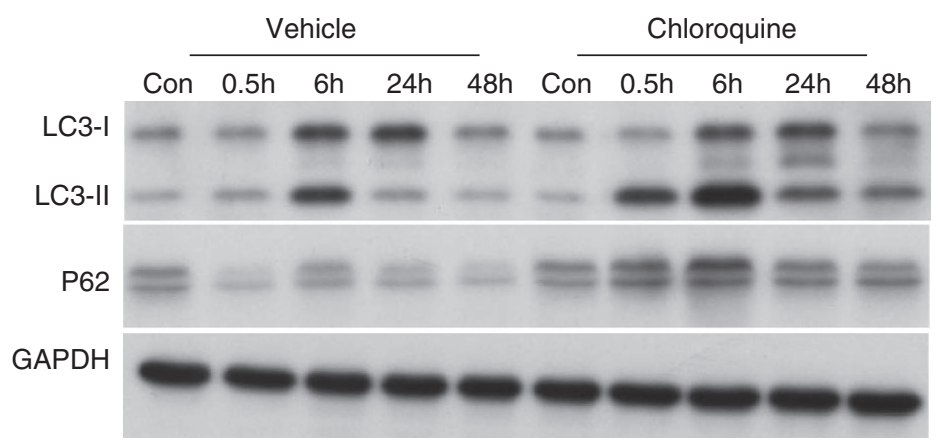

b
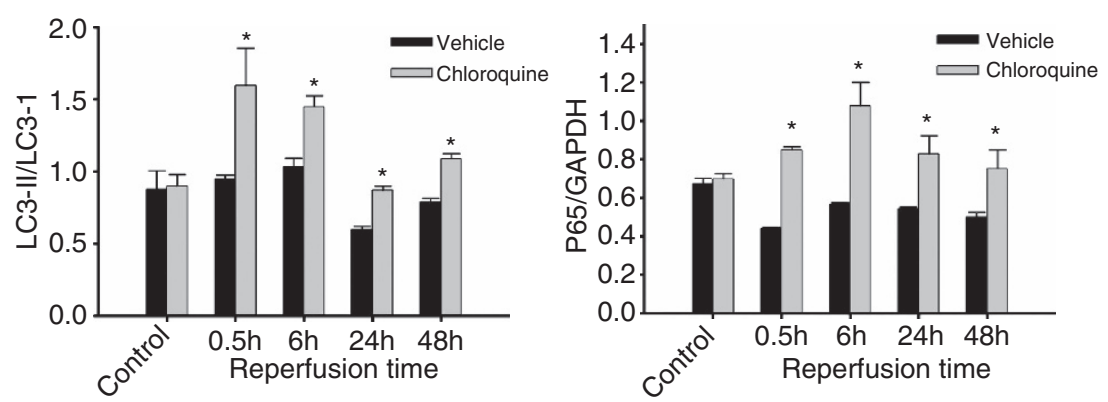

Figure 5 Chloroquine treatment decreased hepatic autophagy following I/R injury. (a) Rats were treated with either chloroquine (60 mg/kg) or vehicle (saline). Liver samples were harvested at $0.5,6,24$ and $48 \mathrm{~h}$ post-reperfusion. Western blot analysis for LC3 and p62 was performed in the ischemic lobes. (b) The gray value of bands was calculated by Image J. Data are shown as mean \pm S.D. $n=6$ per group. ${ }^{*} P<0.05$ compared with vehicle-treated I/R group

a
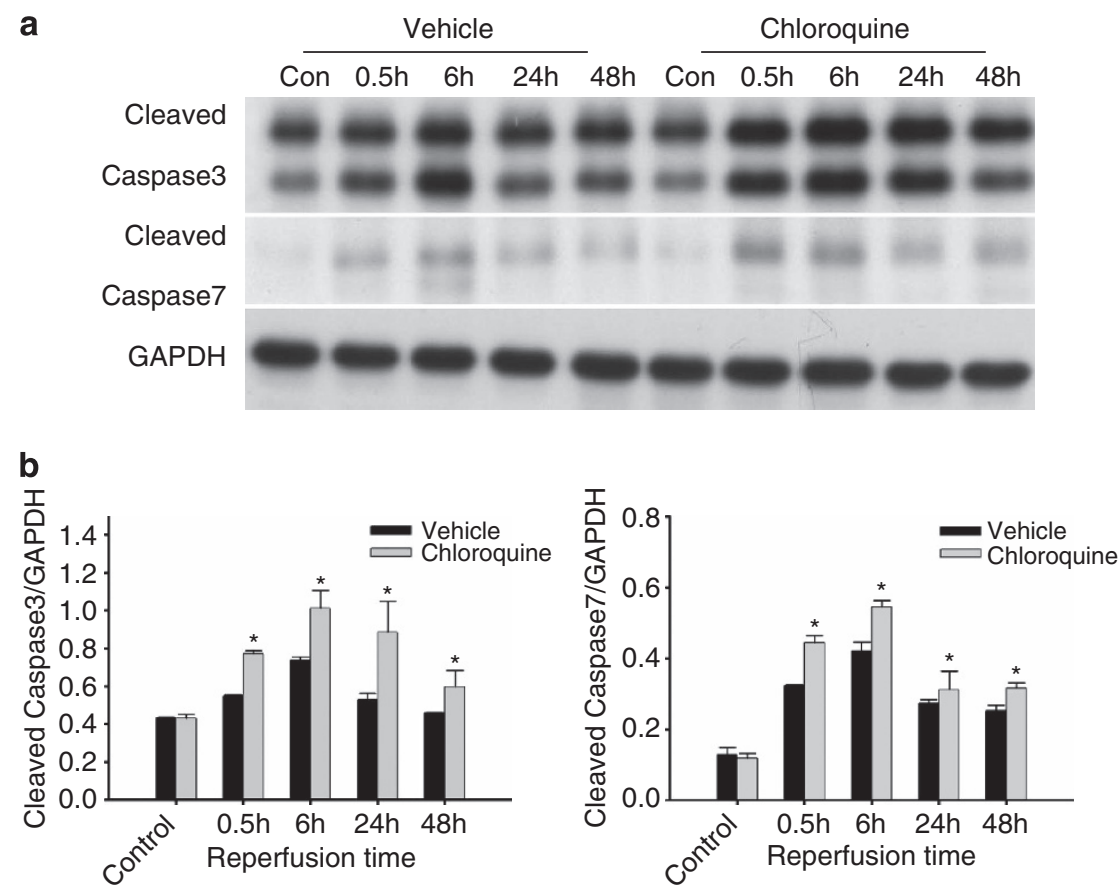

Figure 6 Chloroquine treatment increased hepatic apoptosis following I/R injury. (a) Rats were pre-treated with chloroquine (60 mg/kg) $1 \mathrm{~h}$ before warm ischemia, followed by an additional chloroquine injections at $24 \mathrm{~h}$ after reperfusion. Caspase- 3 and caspase- 7 cleavage was observed by western blots in the ischemic lobes at various time points following reperfusion. (b) The gray value of bands was calculated by Image J. Data are shown as mean \pm S.D. $n=6$ per group. ${ }^{*} P<0.05$ compared with vehicle-treated I/R group

demonstrated that the I/R-induced activation of p38, JNK and ERK was modulated after chloroquine treatment. Of note, I/Rinduced phosphorylation of JNK was inhibited by chloroquine precondition, whereas phosphorylation of ERK was increased by chloroquine precondition. Activation of ERK occurred early in ischemic livers, and ERK activation following redox stress 
Table 2 Characteristics of primers and probes of selected genes

\begin{tabular}{|c|c|c|c|}
\hline Gene & Forward primer & Reverse primer & Probe $^{a}$ \\
\hline $\begin{array}{l}\text { TNF- } \alpha \\
\text { IL-6 } \\
\text { iNOS } \\
\text { IL-1 } \beta \\
\text { HPRT }\end{array}$ & $\begin{array}{l}\text { 5'-TGAACTTCGGGGTGATCG-3' } \\
\text { 5'-CCTGGAGTTTGTGAAGAACAACT-3' } \\
\text { 5'-AAAATGGTTTCCCCCAGTTC-3' } \\
\text { 5'-GCTGACAGACCCCAAAAGAT-3' } \\
\text { 5'-GACCGGTTCTGTCATGTCG-3' }\end{array}$ & $\begin{array}{l}\text { 5'-GGGCTTGTCACTCGAGTTTT-3' } \\
\text { 5'-GGAAGTTGGGGTAGGAAGGA-3' } \\
\text { 5'-CAGCTTGTCCAGGGATTCTG-3' } \\
\text { 5'-AGCTGGATGCTCTCATCTGG-3' } \\
\text { 5'-ACCTGGTTCATCATCACTAATCAC-3' }\end{array}$ & $\begin{array}{c}\# 63 \\
\# 106 \\
\# 95 \\
\# 117 \\
\# 95\end{array}$ \\
\hline
\end{tabular}

aUniversal ProbeLibrary probes

was protective in hepatocytes. ${ }^{33}$ Conversely, JNK activation is a deleterious even in hypoxic hepatocytes via the mitochondrial permeability transition (MPT), and inhibition of JNK activation decreases both necrosis and apoptosis in liver I/R. ${ }^{34}$ Taken together, these data suggest that modulating of MAP kinase activation is also involved in the hepatic protective effects of chloroquine.

To elucidate the molecular basis of chloroquine-mediated injury in the late phase in liver I/R injury, we investigated its effect on the activation of autophagy. Autophagy is a highly conserved regulated cellular process by which cytoplasmic constituents, including organelles are broken down and are recycled through the lysosomal degradation pathway. ${ }^{23,24}$ The execution of autophagy involves a set of evolutionarily conserved autophagy-related gene (Atg) proteins, which are required for the induction of autpophagy, and the generation, maturation, and recycling of autophagosomes. ${ }^{35}$ LC3, a homologue of yeast Atg8, exists in two forms, LC3-I and its proteolytic derivative LC3-II. After synthesis, pro-LC3 is cleaved by Atg4B to expose a C-terminal glycine residue, which represents the cytosolic LC3-I form. LC3-1 is subsequently conjugated with phosphatidylethanolamine to become LC3-II, which localizes on the isolation membrane and the autophagic membranes. As the amount of LC3-II correlates with the number of autophagosomes, LC3-II thus can be used to estimate autophagic activity. ${ }^{36}$ p62 is selectively incorporated into autophagosomes via direct binding to LC3 and is identified as one of the specific substrates that degrade through the autophagy-lysosomal pathway. Thus, the total cellular expression levels of p62 inversely correlate with autophagic activity. ${ }^{36}$ Chloroquine has been recently used to inhibit autophagy in vivo without noticeable side effects. Chloroquine raises the lysosomal $\mathrm{pH}$, which leads to inhibition of lysosome-autophagosome fusion and lysosomal protein degradation. In this study, chloroquine blocked autophagic flux as shown by LC3-II and P62 accumulation and aggravated liver I/R injury after reperfusion. Impaired autophagy leads to the development of MPT in hypoxic hepatocytes. ${ }^{37}$ Onset of the MPT leads to a pH-dependent necrotic cell death as well as apoptotic cell death by releasing proapoptotic proteins factors, which are normally sequestered in the mitochondrial intermembrane space. ${ }^{38-40}$ Indeed, induction of autophagy is associated with attenuation of I/R injury in liver, as demonstrated recently. ${ }^{21,41,42}$ As treatment with chloroquine significantly decreased autophagy and worsened liver injury, we concluded that the decreased autophagy accounted, in part, for the hepatic harmful effects of chloroquine in the late phase after reperfusion.

Apoptotic cell death has a prominent role in the evolution of organ damage following liver $\mathrm{I} / \mathrm{R} .{ }^{43}$ It has been shown that inhibition of autophagy triggers apoptosis. ${ }^{20}$ Apoptosis is defined as suicidal cell death with a particular morphology involving nuclear chromatin condensation, and triggered by activation of either extrinsic (death receptor-mediated) or intrinsic (mitochondrial-associated) death pathways. Recent studies have suggested possible molecular mechanisms for crosstalk between autophagy and apoptosis. ${ }^{44-46} \mathrm{Bcl}-2$ family proteins, the well-characterized apoptosis regulators, has recently become clear that they reduce Beclin 1-mediated autophagy by inhibiting the formation of the Beclin 1-hVps34 PI3K complex and Beclin 1-associated class III PI3K activity. ${ }^{44}$ It has been demonstrated that inhibition of autophagy by chloroquine prevents the fusion of autophagosomes with lysosomes via a disruption in the $\mathrm{pH}$ of acidic vesicles. Thus, the increased cellular burden of autophagosomes has been shown to precede and possibly induce Bax- and mitochondrial-dependent apoptosis. ${ }^{17-20}$ These previous results led us to investigate whether chloroquine could also increase hepatic apoptosis after I/R injury. Our results showed that chloroquine treatment increased apoptotic cell death as indicated by increased I/Rinduced caspase protein cleavage. Together, these data suggest that chloroquine-mediated hepatic harmful effects may involve induction of apoptosis.

In summary, we demonstrate that chloroquine could prevent ischemic liver damage in the early phase, but aggravated liver damage in the late phase of liver I/R injury. The mechanism of protective action of chloroquine in the early phase seems to be related to its ability to decrease inflammatory cytokines and HMGB1 release, and to alter I/R-induced MAP kinase activation. The harmful side effects of chloroquine in the late phase appear to involve its ability to induce apoptosis and to inhibit autophagy. These data suggest that the dual role of chloroquine should be considered when using chloroquine as an inhibitor of inflammation or autophagy in I/R injury.

\section{Materials and Methods}

Experimental design. The experiments were designed to investigate the effects of chloroquine administration on rat hepatic I/R injury. Rats were treated with chloroquine $(60 \mathrm{mg} / \mathrm{kg}$, i.p.) $1 \mathrm{~h}$ before warm ischemia, and they were continuously subjected to a daily chloroquine injection for up to 2 days. Rats were killed $0.5,6,24$ and $48 \mathrm{~h}$ after reperfusion. Liver injury, inflammatory cytokines, hepatic HMGB1 expression and release, MAP kinase activation, autophagy and apoptosis were analyzed.

Animals. Male inbred Lewis rats, purchased from Charles River Laboratories (Sulzfeld, Germany), weighing within $250-320 \mathrm{~g}$, were used in this study. All animals were housed under standard animal care conditions and had free access to water and rat chow ad libitum. All procedures were carried out according to the German Animal Welfare Legislation. 
Chloroquine administration. Chloroquine (Sigma-Aldrich, C6628, St. Louis, MO, USA) was dissolved in normal saline solution. Rats $(n=6$ per group) were pre-treated with chloroquine $(60 \mathrm{mg} / \mathrm{kg}$, i.p.) $1 \mathrm{~h}$ before warm ischemia, followed by an additional chloroquine injections at $24 \mathrm{~h}$ after reperfusion. Animals surviving $0.5,6$ and $24 \mathrm{~h}$ after reperfusion received one injection. Animals surviving $48 \mathrm{~h}$ after reperfusion received two injections.

Partial hepatic warm I/R. Partial hepatic warm I/R model was performed as described previously. ${ }^{8}$ In brief, after opening the abdomen with a transversal incision, interlobular ligaments were dissected. A micro vascular clamp was used to interrupt the arterial and portal venous blood supply to the left lateral and median liver lobes for 60 or $90 \mathrm{~min}$.

Hepatocellular damage assay. To assess liver damage, serum AST and ALT were measured using an Automated Chemical Analyzer (Bayer Advia \#1650; Leverkusen, Germany).

Histopathology. Liver tissue was fixed in $4.5 \%$ buffered formalin and embedded in paraffin. Sections $(4 \mu \mathrm{m})$ were cut and stained with hematoxylineosin. Histological evaluation was performed using a semiquantitative scoring system. ${ }^{47}$

HMGB1 immunohistochemistry. HMGB1 immunohistochemical analysis was performed as described previously. ${ }^{29}$ Briefly, after deparaffinization, rehydration, antigen retrieval and blocking, sections were then incubated with HMGB1 antibody (1:500; Abcam, \#ab18256, Cambridge, UK) for $1 \mathrm{~h}$ at room temperature. Detection was performed by using PowerVision goat-anti-Rabbit-AP (ImmunoLogic, DPVR-110AP, Duiven, the Netherlands) and using Fast-red (Abcam, \#ab64254) as substrate.

Gel electrophoresis and western blotting. Twenty micrograms total protein were separated on $12 \%$ gels by sodium dodecyl sulfate-polyacrylamide gel electrophoresis and transferred to polyvinyldifluoride membranes (GE Healthcare, \#28906837, Buckinghamshire, UK). Antibodies against phosphorylated or total ERK (1: 1000; Cell Signaling Technology, p-ERK, \#4370, ERK, \#4695, Beverly, MA, USA), JNK (1: 1000; Cell Signaling Technology, p-JNK, \#9251, JNK, \#9252) and p38 (1:1000, Cell Signaling Technology, p-p38, \#9211, p38, \#9212); and anti-caspase-3 (1:1000; Cell Signaling Technology, \#9662), caspase-7 (1:1000; Cell Signaling Technology, \#9492), LC3 (1: 1000; Abcam, \#ab48394), SQSTM1/ p62 (1: 1000; Cell Signaling Technology, \#5114), as well as anti-glyceraldehyde3-phosphate dehydrogenase (1:20000; Sigma-Aldrich, \#G9545) were used for western blot analysis. The gray value of bands was calculated by ImageJ $1.43 \mathrm{G}$ (NIH, Bethesda, MD, USA).

Enzyme-linked immunosorbent assay. Serum HMGB1 was determined with a commercial ELISA assay (Shino-Test, ST51011, Kanagawa, Japan). All procedures were performed according to the instructions of the manufacturer.

Quantitative polymerase chain reaction (PCR). Total RNA was extracted from liver tissue using the RNeasy kit (Qiagen, \#74106, Hilden, Germany) and was reverse-transcribed into complementary DNA (CDNA) using the First-Strand cDNA synthesis kit (Invitrogen, \#18080051, Carlsbad, CA, USA). Primers and probes (Table 2) were mixed with Brilliant probe-based QPCR Master Mix (Agilent, \#600881, Santa Clara, CA, USA) and then diluted with distilled deionized $\mathrm{H}_{2} \mathrm{O}$ up to $20 \mu \mathrm{l}$. PCR amplification was performed on an Mx3000P QPCR System (Stratagene, La Jolla, CA, USA). Amplification conditions were: $95^{\circ} \mathrm{C}$ for $5 \mathrm{~min}$, followed by 50 cycles of $95^{\circ} \mathrm{C}$ for $30 \mathrm{~s}$ and $60^{\circ} \mathrm{C}$ for $20 \mathrm{~s}$. Relative quantification of target mRNA expression was calculated and further normalized to hypoxanthine-guanine phosphoribosyltransferase (HPRT).

Statistical analysis. Data were expressed as means \pm S.D. Differences between groups were evaluated for significance by one-way ANOVA analysis. All tests were performed using SigmaStat v3.5 (Systat-Software, Erkrath, Germany). A $P$-value below 0.05 was considered statistically significant.

\section{Conflict of Interest}

The authors declare no conflict of interest.
Acknowledgements. This project was supported by the German Federal Ministry for Education and Research (BMBF) Virtual Liver Network. We thank Stephanie Lange for excellent animal care.

1. Fondevila C, Busuttil RW, Kupiec-Weglinski JW. Hepatic ischemia/reperfusion injury-a fresh look. Exp Mol Pathol 2003; 74: 86-93.

2. Selzner N, Rudiger $H$, Graf R, Clavien PA. Protective strategies against ischemic injury of the liver. Gastroenterology 2003; 125: 917-936.

3. Vardanian AJ, Busuttil RW, Kupiec-Weglinski JW. Molecular mediators of liver ischemia and reperfusion injury: a brief review. Mol Med 2008; 14: 337-345.

4. Teoh NC, Farrell GC. Hepatic ischemia reperfusion injury: pathogenic mechanisms and basis for hepatoprotection. J Gastroenterol Hepatol 2003; 18: 891-902.

5. Montalvo-Jave EE, Escalante-Tattersfield T, Ortega-Salgado JA, Pina E, Geller DA. Factors in the pathophysiology of the liver ischemia-reperfusion injury. J Surg Res 2008; 147: 153-159.

6. Steininger R, Roth E, Fugger R, Winkler S, Langle F, Grunberger T et al. Transhepatic metabolism of TNF-alpha, IL-6, and endotoxin in the early hepatic reperfusion period after human liver transplantation. Transplantation 1994; 58: 179-183.

7. Colletti LM, Kunkel SL, Walz A, Burdick MD, Kunkel RG, Wilke CA et al. The role of cytokine networks in the local liver injury following hepatic ischemia/reperfusion in the rat. Hepatology 1996; 23: 506-514.

8. Liu A, Dirsch O, Fang H, Sun J, Jin H, Dong W et al. HMGB1 in ischemic and non-ischemic liver after selective warm ischemia/reperfusion in rat. Histochem Cell Biol 2011; 135: 443-452.

9. Tsung A, Sahai R, Tanaka H, Nakao A, Fink MP, Lotze MT et al. The nuclear factor HMGB1 mediates hepatic injury after murine liver ischemia-reperfusion. J Exp Med 2005; 201: 1135-1143.

10. Baird JK.. Effectiveness of antimalarial drugs. N Engl J Med 2005; 352: 1565-1577.

11. Hong Z, Jiang Z, Liangxi W, Guofu D, Ping L, Yongling L et al. Chloroquine protects mice from challenge with $\mathrm{CpG} O D N$ and LPS by decreasing proinflammatory cytokine release. Int Immunopharmacol 2004; 4: 223-234.

12. Karres I, Kremer JP, Dietl I, Steckholzer U, Jochum M, Ertel W. Chloroquine inhibits proinflammatory cytokine release into human whole blood. Am J Physiol 1998; 274: R1058-R1064.

13. Lim EJ, Lee SH, Lee JG, Kim JR, Yun SS, Baek SH et al. Toll-like receptor 9 dependent activation of MAPK and NF-KB is required for the CpG ODN-induced matrix metalloproteinase-9 expression. Exp Mol Med 2007; 39: 239-245.

14. Yasuda H, Leelahavanichkul A, Tsunoda S, Dear JW, Takahashi Y, Ito S et al. Chloroquine and inhibition of Toll-like receptor 9 protect from sepsis-induced acute kidney injury. Am J Physiol Renal Physiol 2008; 294: F1050-F1058.

15. Jiang M, Liu K, Luo J, Dong Z. Autophagy is a renoprotective mechanism during in vitro hypoxia and in vivo ischemia-reperfusion injury. $A m$ J Pathol 2010; 176: 1181-1192.

16. Hoshino A, Matoba S, Iwai-Kanai E, Nakamura H, Kimata M, Nakaoka M et al. p53-TIGAR axis attenuates mitophagy to exacerbate cardiac damage after ischemia. J Mol Cell Cardiol 2012; 52: 175-184.

17. Shacka JJ, Klocke BJ, Shibata M, Uchiyama Y, Datta G, Schmidt RE et al. Bafilomycin $A 1$ inhibits chloroquine-induced death of cerebellar granule neurons. Mol Pharmacol 2006; 69: 1125-1136.

18. Kim EL, Wustenberg R, Rubsam A, Schmitz-Salue C, Warnecke G, Bucker EM et al. Chloroquine activates the p53 pathway and induces apoptosis in human glioma cells. Neuro Oncol 2010; 12: 389-400.

19. Zaidi AU, McDonough JS, Klocke BJ, Latham CB, Korsmeyer SJ, Flavell RA et al. Chloroquine-induced neuronal cell death is $\mathrm{p} 53$ and Bcl-2 family-dependent but caspase-independent. J Neuropathol Exp Neurol 2001; 60: 937-945.

20. Boya P, Gonzalez-Polo RA, Casares N, Perfettini JL, Dessen P, Larochette N et al. Inhibition of macroautophagy triggers apoptosis. Mol Cell Biol 2005; 25: 1025-1040.

21. Rautou PE, Mansouri A, Lebrec D, Durand F, Valla D, Moreau R. Autophagy in liver diseases. J Hepatol 2010; 53: 1123-1134.

22. Schmid D, Dengjel J, Schoor O, Stevanovic S, Munz C. Autophagy in innate and adaptive immunity against intracellular pathogens. J Mol Med (Berl) 2006; 84: 194-202.

23. Klionsky DJ, Emr SD. Autophagy as a regulated pathway of cellular degradation. Science 2000; 290: 1717-1721.

24. Glick D, Barth S, Macleod KF. Autophagy: cellular and molecular mechanisms. $J$ Pathol 2010; 221: 3-12.

25. Bradham CA, Stachlewitz RF, Gao W, Qian T, Jayadev S, Jenkins G et al. Reperfusion after liver transplantation in rats differentially activates the mitogen-activated protein kinases. Hepatology 1997; 25: 1128-1135.

26. Janku F, McConkey DJ, Hong DS, Kurzrock R. Autophagy as a target for anticancer therapy. Nat Rev Clin Oncol 2011; 8: 528-539.

27. Sun S, Rao NL, Venable J, Thurmond R, Karlsson L. TLR7/9 antagonists as therapeutics for immune-mediated inflammatory disorders. Inflamm Allergy Drug Targets 2007; 6: 223-235.

28. Lee VG, Johnson ML, Baust J, Laubach VE, Watkins SC, Billiar TR. The roles of iNOS in liver ischemia-reperfusion injury. Shock 2001; 16: 355-360. 
29. Liu A, Dirsch O, Fang H, Dong W, Jin H, Huang $\mathrm{H}$ et al. HMGB1 translocation and expression is caused by warm ischemia reperfusion injury, but not by partial hepatectomy in rats. Exp Mol Pathol 2011; 91: 502-508.

30. Liu A, Jin H, Dirsch O, Deng M, Huang H, Brocker-Preuss $M$ et al. Release of danger signals during ischemic storage of the liver: a potential marker of organ damage? Mediators Inflamm 2010; 2010: 436145

31. Kono M, Tatsumi K, Imai AM, Saito K, Kuriyama T, Shirasawa H. Inhibition of human coronavirus 229E infection in human epithelial lung cells (L132) by chloroquine: involvement of p38 MAPK and ERK. Antiviral Res 2008; 77: 150-152.

32. Yi AK, Krieg AM. Rapid induction of mitogen-activated protein kinases by immune stimulatory CpG DNA. J Immunol 1998; 161: 4493-4497.

33. Czaja MJ, Liu H, Wang Y. Oxidant-induced hepatocyte injury from menadione is regulated by ERK and AP-1 signaling. Hepatology 2003; 37: 1405-1413.

34. Uehara T, Bennett B, Sakata ST, Satoh Y, Bilter GK, Westwick JK et al. JNK mediates hepatic ischemia reperfusion injury. J Hepatol 2005; 42: 850-859.

35. Mizushima N, Yoshimori T, Ohsumi Y. The role of Atg proteins in autophagosome formation. Annu Rev Cell Dev Biol 2011; 27: 107-132.

36. Mizushima N, Yoshimori T, Levine B. Methods in mammalian autophagy research. Cell 2010; 140: 313-326.

37. Kim JS, Nitta T, Mohuczy D, O'Malley KA, Moldawer LL, Dunn WA Jr. et al. Impaired autophagy: a mechanism of mitochondrial dysfunction in anoxic rat hepatocytes. Hepatology 2008; 47: 1725-1736.

38. Qian T, Nieminen AL, Herman B, Lemasters JJ. Mitochondrial permeability transition in $\mathrm{pH}$ dependent reperfusion injury to rat hepatocytes. Am J Physiol 1997; 273: C1783-C1792.

39. Kim JS, He L, Qian T, Lemasters JJ. Role of the mitochondrial permeability transition in apoptotic and necrotic death after ischemia/reperfusion injury to hepatocytes. Curr Mol Med 2003; 3: 527-535
40. Kim JS, Qian T, Lemasters JJ. Mitochondrial permeability transition in the switch from necrotic to apoptotic cell death in ischemic rat hepatocytes. Gastroenterology 2003; 124: 494-503.

41. Esposti DD, Domart MC, Sebagh M, Harper F, Pierron G, Brenner C et al. Autophagy is induced by ischemic preconditioning in human livers formerly treated by chemotherapy to limit necrosis. Autophagy 2010; 6: 172-174.

42. Yin XM, Ding WX, Gao W. Autophagy in the liver. Hepatology 2008; 47: 1773-1785.

43. Malhi H, Gores GJ, Lemasters JJ. Apoptosis and necrosis in the liver: a tale of two deaths? Hepatology 2006; 43: S31-S44.

44. Zhou F, Yang Y, Xing D. Bcl-2 and Bcl-xL play important roles in the crosstalk between autophagy and apoptosis. FEBS J 2011; 278: 403-413.

45. Levine B, Sinha S, Kroemer G. Bcl-2 family members: dual regulators of apoptosis and autophagy. Autophagy 2008; 4: 600-606

46. Rubinstein AD, Eisenstein M, Ber Y, Bialik S, Kimchi A. The autophagy protein Atg12 associates with antiapoptotic Bcl-2 family members to promote mitochondrial apoptosis. Mol Cell 2011; 44: 698-709.

47. Fang H, Liu A, Sun J, Kitz A, Dirsch O, Dahmen U. Granulocyte colony stimulating factor induces lipopolysaccharide (LPS) sensitization via upregulation of LPS binding protein in rat. PLoS One 2013; 8: e56654.

(c) (i) () $\Theta$ Cell Death and Disease is an open-access journal By ${ }^{2}$ published by Nature Publishing Group. This work is licensed under a Creative Commons Attribution-NonCommercialNoDerivs 3.0 Unported License. To view a copy of this license, visit http://creativecommons.org/licenses/by-nc-nd/3.0/ 